\title{
Optimal Boundary Control \& Estimation of Diffusion-Reaction PDEs
}

\author{
Scott J. Moura and Hosam K. Fathy
}

\begin{abstract}
This paper considers the optimal control and optimal estimation problems for a class of linear parabolic diffusion-reaction partial differential equations (PDEs) with actuators and sensors at the boundaries. Diffusion-reaction PDEs with boundary actuation and sensing arise in a multitude of relevant physical systems (e.g. magneto-hydrodynamic flows, chemical reactors, and electrochemical conversion devices). We formulate both the control and estimation problems using finite-time optimal control techniques, where the key results represent first order necessary conditions for optimality. Specifically, the time-varying state-feedback and observer gains are determined by solving Riccati-type PDEs. These results are analogous to the Riccati differential equations seen in linear quadratic regulator and optimal estimator results. In this sense, this paper extends LQR and optimal estimation results for finite-dimensional systems to infinite-dimensional systems with boundary actuation and sensing. These results are unique in two important ways. First, the derivations completely avoid discretization until the implementation stage. Second, they bypass formulating infinite-dimensional systems on an abstract Hilbert space and applying semigroup theory. Instead, Riccati equations are derived by applying weak-variations directly on the PDEs. Simulation examples and comparative analyses to backstepping are included for demonstration purposes.
\end{abstract}

\section{INTRODUCTION}

In this paper we examine the linear quadratic regulator and optimal estimator problems for linear parabolic diffusionreaction partial differential equations (PDEs) with actuation and sensing at the boundaries. A broad spectrum of physical engineering systems exhibit diffusion-reaction dynamics, e.g. structural acoustics [1], fixed-bed reactors [2], multi-agent coordination control [3], and stock investment models [4]. A subset of these systems limit control and sensing to the boundaries, such as thermal/fluid flows [5], cardiovascular systems [6], chemical reactors [7], and advanced batteries [8]-[10]. Optimal control and estimation of these PDE systems is particularly challenging since actuation and sensing are limited to the boundary and the dynamics are notably more complex than ODE systems. Motivated by this fact, this paper's overall goal is to develop optimal boundary control and estimation algorithms for parabolic PDE systems. We specifically focus on diffusion-reaction PDEs with Dirichlet actuation and anti-collocated sensors and actuators. Results are also limited to finite-time horizons. The approaches

This work was supported in part by the National Science Foundation Graduate Research Fellowship Program.

S. J. Moura is with the Department of Mechanical Engineering, University of Michigan, Ann Arbor, MI 48109-2133 USA (e-mail: s jmoura@umich. edu)

H. K. Fathy (corresponding author) is with the Department of Mechanical and Nuclear Engineering, Penn State University, University Park, PA 16802 , USA (e-mail: hkf2 @psu . edu) presented here, however, are general to different system configurations.

Optimal control of PDE systems has a rich history (see [11] for a particularly excellent survey of results). One can generally place this research into two categories. The first category projects the PDEs onto a finite-dimensional subspace to render the system into a series of ordinary differential equations (ODEs). This enables them to apply classical optimal control results [12]-[14]. Yet this method necessarily couples the control problem with the projection technique. The second category of research applies semigroup theory to represent PDE systems as ODE systems over Hilbert spaces. From here the classical optimal control results are extended into infinite-dimensional systems [15]-[17]. Ultimately, these techniques produce so-called operator Riccati equations which have similarities to the results presented here.

The main goal of this paper is to bridge the gap between the aforementioned two categories. Namely, we wish to separate the discretization techniques from the controller/estimator design by maintaining the analysis in the infinite-dimensional domain. Secondly, we bypass semigroup theory and the associated issues with solving operator Riccati equations by applying optimization theory directly to the PDEs. Hence, this paper adds two important and original contributions. First, we derive the first order necessary conditions for optimality of a quadratic cost criterion. These conditions manifest themselves as coupled PDEs for the states and co-states with split initial/final conditions. We then postulate a time-varying feedback control law form, where the feedback transformation operator satisfies a Riccati-like partial differential equation. Second, we solve the optimal estimator problem in a similar manner by deriving first-order necessary conditions for a quadratic cost criteria that eventually produces a dual-Riccati partial differential equation. In both cases the results are independent of the numerical scheme used to implement the algorithms. Moreover, the conditions governing the optimal linear operators are PDE systems themselves and therefore straight-forward to solve. Finally, the Riccati PDEs have similarities to the operator Riccati equations reported by past researchers (e.g., [15] [17]), yet are derived using a completely different technique, namely weak-variations. These features provide an elegant and accessible method for optimal boundary control and estimation of parabolic PDE systems.

The remainder of the paper is organized as follows: Section II presents preliminary mathematics and notation used to extend linear optimal control and estimation to infinitedimensional systems. Section III presents linear quadratic 
regulator results for diffusion-reaction PDEs. This includes the open loop control problem, state-feedback, and numerical examples. Section IV presents the linear estimator results for diffusion-reaction PDEs with numerical examples. Finally, Section V summarizes the key results of the paper.

\section{MATHEMATICAL PRELIMINARIES \& NOTATION}

Here, we introduce some preliminary mathematics useful for extending linear optimal control to infinite-dimensional systems.

$$
\begin{array}{ll}
\text { Linear Operator: } & A(f(x)):=\int_{0}^{1} A(x, y) f(y) d y \\
\text { Inner Product: } & \langle f(x), g(x)\rangle:=\int_{0}^{1} f(x) g(x) d x \\
\text { Sifting Property of the } & \int \delta(y) f(y)=f(0) \\
\text { Dirac-delta function: } & \\
\text { Derivative of the } & \int \delta^{\prime}(y) f(y) d y=-\int \delta(y) \frac{\partial f}{\partial y} d y \\
\text { Dirac-delta function: } &
\end{array}
$$

Subscripts denote partial derivatives with respect to the notated variable. For example $u_{t}=\partial u / \partial t$ and $\lambda_{x}=\partial \lambda / \partial x$. Arguments to spatially/temporally dependent variables are listed in order of space then time. Arguments are dropped when they are clear from the context. Finally, some proofs are abbreviated to highlight only key steps of the derivations, due to space limitations. The full details will be provided in a future journal publication.

\section{LINEAR QUADRATIC REGULATOR}

\section{A. Problem Statement}

Consider the following class of linear parabolic diffusionreaction partial differential equations:

$$
\begin{aligned}
u_{t}(x, t) & =u_{x x}(x, t)+c u(x, t) \\
u_{x}(0, t) & =0 \\
u(1, t) & =U(t) \\
u(x, 0) & =u_{0}(x)
\end{aligned}
$$

The first term in (1) represents diffusion and the second term models linear reaction phenomena. Non-unity diffusivity coefficients, lengths, input gains, etc. can be accounted for by non-dimensionalizing the system into the form given above. Suppose we can control the boundary value $u(1, t)$ (Dirichlet control) and nothing else. Moreover, suppose we have noiseless measurements of the state available throughout the spatial domain. Our goal is to develop a state-feedback controller that optimally regulates the system to the origin. Specifically, minimize the following quadratic objective over a finite time-horizon:

$$
\begin{aligned}
J= & \frac{1}{2} \int_{0}^{T}\left[\langle u(x, t), Q(u(x, t))\rangle+R U^{2}(t)\right] d t+ \\
& \frac{1}{2}\left\langle u(x, T), P_{f}(u(x, T))\right\rangle
\end{aligned}
$$

The symbols $Q, R$, and $P_{f}$ are weighting kernels that respectively weight the state, control, and terminal state of the closed loop system. Note that $R>0$ should be satisfied to ensure bounded control signals. First, we derive the necessary conditions for optimality of the open-loop finite-horizon control problem using weak variations. Instead of obtaining coupled ordinary differential equations with split initial conditions for finite-dimensional LQR, we obtain coupled partial differential equations with split initial conditions. Next, we postulate the open-loop control signal can be written in state-feedback form and derive the associated Ricatti equation for the feedback linear operator. This Ricatti equation is a 2-D spatial, 1-D temporal PDE. We then demonstrate the LQR result in simulation and compare it to the backstepping approach.

\section{B. Open Loop Control}

We start by deriving the first order necessary conditions for the open loop finite-time horizon problem.

Theorem 1: Consider the linear diffusion-reaction PDE described by (1)-(4) defined on the finite-time horizon $t \in$ $[0, T]$ with quadratic cost criteria (5). Let $u^{*}(x, t), U^{*}(t)$, and $\lambda(x, t)$ respectively denote the optimal state, control, and costate that minimize the quadratic cost. Then the first order necessary conditions for optimality are:

$$
\begin{aligned}
u_{t}^{*}(x, t) & =u_{x x}^{*}(x, t)+c u^{*}(x, t) \\
-\lambda_{t}(x, t) & =\lambda_{x x}+c \lambda(x, t)+Q\left(u^{*}(x, t)\right)
\end{aligned}
$$

with boundary conditions

$$
\begin{aligned}
& u_{x}^{*}(0, t)=0 \quad u^{*}(1, t)=U^{*}(t) \\
& \lambda_{x}(0, t)=0 \quad \lambda(1, t)=0
\end{aligned}
$$

and split initial/final conditions

$$
u^{*}(x, 0)=u_{0}(x) \quad \lambda(x, T)=P_{f}\left(u^{*}(x, T)\right)
$$

and the optimal control input is

$$
U^{*}(t)=\frac{1}{R} \lambda_{x}(1, t)
$$

Proof: The necessary conditions are derived via weak variations [18]. Suppose $u^{*}(x, t)$ and $U^{*}(t)$ are the optimal state and control inputs. Let $u(x, t)=u^{*}(x, t)+\epsilon \delta u(x, t)$, $U(t)=U^{*}(t)+\epsilon \delta U(t)$ and $\delta u(x, 0)=0$ represent perturbations from the optimal solutions. Then the cost is

$$
\begin{aligned}
& J\left(u^{*}+\epsilon \delta u, U^{*}+\epsilon \delta U\right)= \\
& \quad \frac{1}{2} \int_{0}^{T}\left[\left\langle u^{*}+\epsilon \delta u, Q\left(u^{*}+\epsilon \delta u\right)\right\rangle+R\left(U^{*}+\epsilon \delta U\right)^{2}\right] d t \\
& +\frac{1}{2}\left\langle u^{*}(T)+\epsilon \delta u(T), P_{f}\left(u^{*}(T)+\epsilon \delta u(T)\right)\right\rangle
\end{aligned}
$$

Define the following quantity

$$
\begin{aligned}
g(\epsilon) & := \\
& \frac{1}{2} \int_{0}^{T}\left[\left\langle u^{*}+\epsilon \delta u, Q\left(u^{*}+\epsilon \delta u\right)\right\rangle+R\left(U^{*}+\epsilon \delta U\right)^{2}\right] d t \\
& +\frac{1}{2}\left\langle u^{*}(T)+\epsilon \delta u(T), P_{f}\left(u^{*}(T)+\epsilon \delta u(T)\right)\right\rangle \\
& +\int_{0}^{T}\left\langle\lambda(x), u_{x x}^{*}+\epsilon \delta u_{x x}+c u^{*}+\epsilon c \delta u\right\rangle d t \\
& -\int_{0}^{T}\left\langle\lambda(x), \frac{\partial}{\partial t}\left(u^{*}+\epsilon \delta u\right)\right\rangle d t
\end{aligned}
$$


where the last term equals zero and accounts for the system dynamics constraint (1) in a Lagrangian form. Then the necessary condition for optimality is $d g(\epsilon) /\left.d \epsilon\right|_{\epsilon=0}=0$. Differentiating $g(\epsilon)$ gives:

$$
\begin{aligned}
\frac{d g}{d \epsilon}(\epsilon) & =\int_{0}^{T}\left[\left\langle\delta u, Q\left(u^{*}+\epsilon \delta u\right)\right\rangle+R\left(U^{*}+\epsilon \delta U\right) \delta U\right] d t \\
& +\left\langle\delta u(T), P_{f}\left(u^{*}(T)+\epsilon \delta u(T)\right)\right\rangle \\
& +\int_{0}^{T}\left\langle\lambda(x), \delta u_{x x}+c \delta u-\frac{\partial}{\partial t}(\delta u)\right\rangle d t
\end{aligned}
$$

We simplify the third term further by applying integration by parts. Specifically, one can show that

$$
\begin{aligned}
\left\langle\lambda(x), \delta u_{x x}(x)\right\rangle= & \lambda(1) \delta u_{x}(1)-\lambda(0) \delta u_{x}(0) \\
& -\lambda_{x}(1) \delta u(1)+\lambda_{x}(0) \delta u(0)
\end{aligned}
$$

By applying the boundary conditions for $\delta u(x, t)$ we note that $\delta u_{x}(0, t)=0$ and $\delta u(1, t)=\delta U(t)$, resulting in

$$
\left\langle\lambda(x), \delta u_{x x}(x)\right\rangle=\lambda(1) \delta u_{x}(1)-\lambda_{x}(1) \delta U(t)+\lambda_{x}(0) \delta u(0)
$$

One can also use integration by parts to show that:

$$
\begin{aligned}
\int_{0}^{T}\left\langle\lambda(x), \delta u_{x x}(x)\right\rangle d t= & \langle\lambda(T), \delta u(T)\rangle-\langle\lambda(0), \delta u(0)\rangle \\
& -\int_{0}^{T}\left\langle\lambda_{t}, \delta u\right\rangle d t
\end{aligned}
$$

Note that $\delta u(x, 0)=0$ by definition. Therefore

$$
\int_{0}^{T}\left\langle\lambda(x), \delta u_{x x}(x)\right\rangle d t=\langle\lambda(T), \delta u(T)\rangle-\int_{0}^{T}\left\langle\lambda_{t}, \delta u\right\rangle d t
$$

At this point we plug (16) and (18) into (14) and collect like perturbation terms

$$
\begin{aligned}
\frac{d g}{d \epsilon}(\epsilon)= & \int_{0}^{T}\left[\left\langle Q\left(u^{*}+\epsilon \delta u\right), \delta u\right\rangle+\left\langle\lambda_{x x}+c \lambda+\lambda_{t}, \delta u\right\rangle\right] d t \\
& +\int_{0}^{T}\left[R\left(U^{*}+\epsilon \delta U\right)-\lambda_{x}(1)\right] \delta U d t \\
& +\int_{0}^{T}\left[\lambda(1) \delta u_{x}(1)+\lambda_{x}(0) \delta u(0)\right] d t \\
& +\left\langle P_{f}\left(u^{*}(T)+\epsilon \delta u(T)\right), \delta u(T)\right\rangle
\end{aligned}
$$

Now we evaluate the previous expression at $\epsilon=0$ and set it equal to zero.

$$
\begin{aligned}
\left.\frac{d g}{d \epsilon}(\epsilon)\right|_{\epsilon=0}= & \int_{0}^{T}\left[\left\langle Q\left(u^{*}\right)+\lambda_{x x}+c \lambda+\lambda_{t}, \delta u\right\rangle\right] d t \\
& +\int_{0}^{T}\left[R U^{*}-\lambda_{x}(1)\right] \delta U d t \\
& +\int_{0}^{T}\left[\lambda(1) \delta u_{x}(1)+\lambda_{x}(0) \delta u(0)\right] d t \\
& +\left\langle P_{f}\left(u^{*}(T)\right), \delta u(T)=0\right.
\end{aligned}
$$

For the previous equation to hold true for all arbitrary $\delta u(x, t), \delta U(t), \delta u(x, T)$, the following conditions must hold:

$$
\begin{aligned}
-\lambda_{t}(x, t) & =\lambda_{x x}(x, t)+c \lambda(x, t)+Q\left(u^{*}(x, t)\right) \\
\lambda_{x}(0, t) & =0 \quad \lambda(1, t)=0 \\
\lambda(x, T) & =P_{f}\left(u^{*}(x, T)\right) \\
U^{*}(t) & =\frac{1}{R} \lambda_{x}(1, t)
\end{aligned}
$$

These conditions respectively represent the dynamics, boundary conditions, final condition for the co-state, and the optimal boundary control. Coupled together with the plant dynamics, these conditions represent the first order necessary conditions of optimality, which completes the proof.

Remark 2: In general weak-variations provide the necessary conditions for optimality and the Hamilton-JacobiBellman equation provides the sufficient condition for optimality. However, both methods provide necessary and sufficient conditions when considering a strictly convex cost functional, as we do in this paper [15].

\section{State-Feedback Control}

Now let us consider the state-feedback problem. That is, let us postulate that the co-state $\lambda$ is related to the states according to the time-varying linear transformation:

$$
\lambda(x, t)=P^{t}(u(x, t))=\int_{0}^{1} P(x, y, t) u(y, t) d y
$$

The superscript on $P^{t}$ indicates the linear operator is timedependent.

Theorem 3: The optimal control in state-feedback form is:

$$
U^{*}(t)=\frac{1}{R}\left[\frac{\partial}{\partial x} P^{t}\left(u^{*}(x, t)\right)\right]_{x=1}
$$

where the time-varying linear transformation $P^{t}$ must satisfy the following Riccati-like PDE:

$$
\begin{aligned}
& -P_{t}=P_{x x}+P_{y y}+2 c P+Q-\frac{1}{R} P_{y}(x, 1) P_{x}(1, y) \\
& \text { with boundary conditions }
\end{aligned}
$$

with boundary conditions

$$
P_{x}(0, y, t)=P(1, y, t)=P(x, 0, t)=P(x, 1, t)=0
$$

and final condition

$$
P(x, y, T)=P_{f}(x, y)
$$

Proof: The proof consists of evaluating each $\lambda$ term in (7), (9), and (10) using the postulated form in (25). Two boundary conditions for the Riccati PDE result directly from (9) and the other two arise from integration by parts.

Note that the Riccati-like PDE in (27)-(29) is quadratic and must be evaluated backwards in time, like the Riccati differential equation for finite-dimensional systems.

\section{Simulation Example}

In this section we present simulation examples of the linear quadratic regulator. Until now the presented results are independent of the specific numerical scheme used to implement the controller. That is, the theory is general to any simulation technique, e.g. finite difference, finite element, or spectral methods to name a few. In this paper we use 
(a) LQR

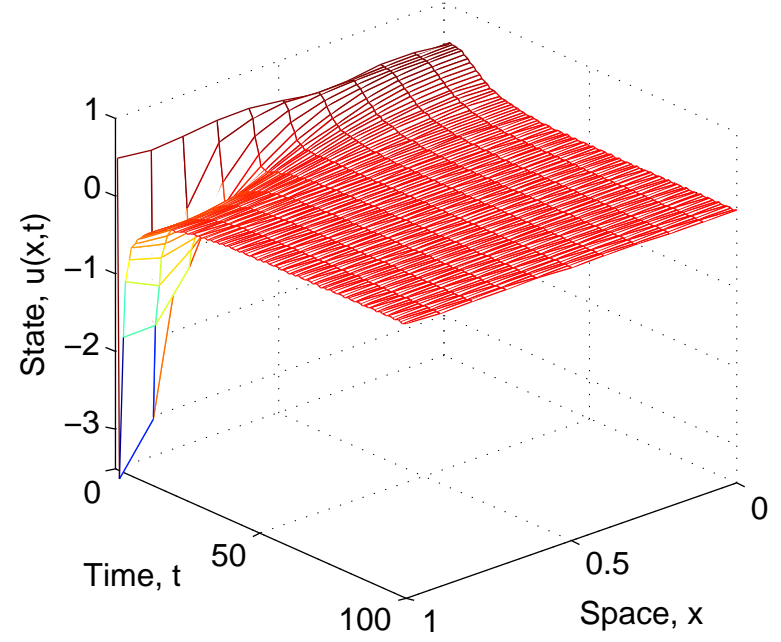

(b) Backstepping

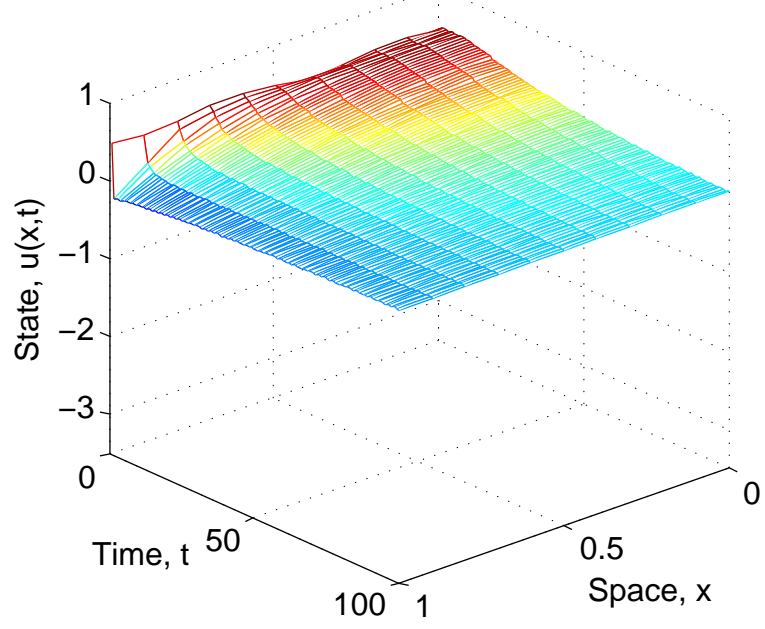

Fig. 1. Simulation example of state trajectories for the (a) LQR and (b) backstepping controllers.

TABLE I

PARAMETER VALUES FOR LQR SimULATION EXAMPLES

\begin{tabular}{ll}
\hline \hline Parameter & Value \\
\hline \hline Reaction coefficient & $c=1$ \\
State weight kernel & $Q(x, y)=10^{-2} \cdot I(x, y)$ \\
Control weight kernel & $R=1$ \\
Final state weight kernel & $P_{f}(x, y)=I(x, y)$ \\
Initial state & $u(x, 0)=0.5+0.05 \sin (2 \pi \times 2 x)+$ \\
Time Horizon & $0.02 \sin (2 \pi \times 5 x)-0.01 \sin (2 \pi \times 21 x)$ \\
\hline \hline
\end{tabular}

the Crank-Nicolson method to solve PDEs [19]. Throughout these examples we consider the class of linear parabolic partial differential equation systems described by (1)-(4).

Here we demonstrate the linear quadratic regulator results, where the optimal control is given by (26), and the timevarying linear operator $P^{t}$ is the unique solution of the Riccati PDE (27)-(29). The parameters for this example are shown in Table I. Note the state is initialized to an arbitrary non-zero initial condition. The evolution of the state for the closed-loop system is displayed in Fig. 1(a), which settles to the origin by the end of the time horizon. This can also be seen in the tracking error provided in Fig. 2(a). The boundary control input is displayed in Fig. 2(b), which decays to zero as the state reaches the origin. The initial boundary control value exhibits a sharp spike because it corresponds to the initial state at the boundary.

\section{E. Comparison to Backstepping}

In this section we compare the LQR results to a wellestablished boundary control technique - backstepping [20]. The heart of backstepping involves the design of a linear Volterra transformation that forces the dynamics to an exponentially stable target system. The target system is usually the heat equation $\left(w_{t}=w_{x x}\right)$ with zero boundary conditions $\left(w_{x}(0, t)=w(1, t)=0\right)$, and is the one we will consider in this paper. Like the methods presented here, backstepping ultimately involves the solution of a PDE related to the gain kernels. It is important to note that the backstepping gains are static in time, where as the optimal regulator and estimator gains for finite-time problems are time-varying.

For the state-feedback control problem, it has been shown in [21] that the backstepping control input is given by

$$
U(t)=\langle k(1, y), u(x, t)\rangle=\int_{0}^{1} k(1, y) u(y, t) d y
$$

where the gain kernel $k(x, y)$ is related to the solution of the following Klein-Gordon hyperbolic PDE

$$
\begin{aligned}
k_{x x}(x, y)-k_{y y}(x, y) & =c k(x, y) \\
k(x, x) & =-\frac{1}{2} c x \\
k_{y}(x, 0) & =0
\end{aligned}
$$

solved on the region $\{x, y: 0<y<x<1\}$. Using a summation of successive approximation series, one can show that the solution to this PDE is

$$
k(x, y)=-c x \frac{I_{1}\left(\sqrt{c\left(x^{2}-y^{2}\right)}\right)}{\sqrt{c\left(x^{2}-y^{2}\right)}}
$$

where $I_{1}$ is the first-order modified Bessel function of the first kind. Then the backstepping control input is given in state-feedback form as

$$
U(t)=-\int_{0}^{1} c \frac{I_{1}\left(\sqrt{c\left(1-y^{2}\right)}\right)}{\sqrt{c\left(1-y^{2}\right)}} u(y) d y
$$

The state-trajectories for the backstepping controller are shown in Fig. 1(b), adjacent to the LQR controller results. In comparison, one can see that $\mathrm{LQR}$ is more aggressive than backstepping in forcing the state to the origin. This fact is also seen in the $\mathrm{L}^{2}$-norm of tracking error plotted in Fig. 


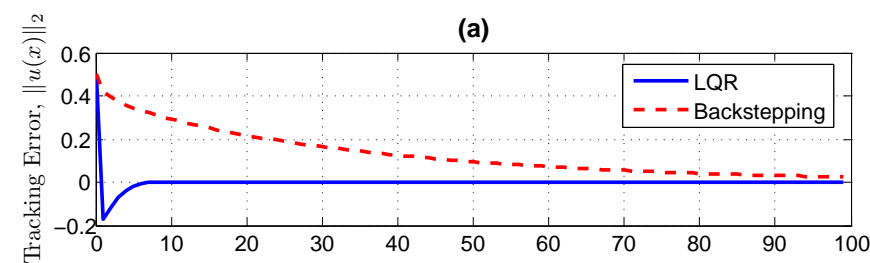

(b)

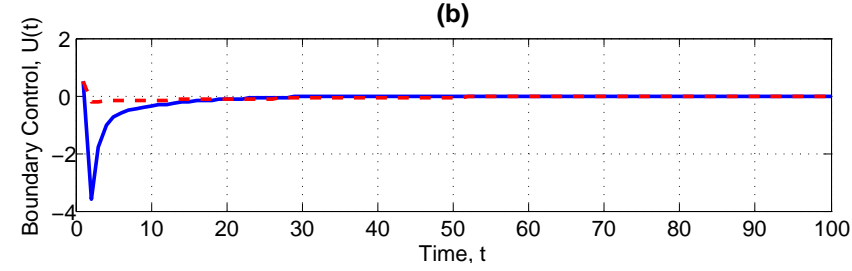

Fig. 2. (a) Tracking error and (b) boundary control inputs for LQR and backstepping controllers

2(a). The faster convergence rate for $\mathrm{LQR}$ is due to higher magnitude boundary control inputs, as seen in Fig. 2(b). One may increase the convergence speed for backstepping by modifying the target system to have a diffusion coefficient whose value is greater than one, for example. Regardless, the LQR controller performs well compared to the battle-tested backstepping approach. The critical design difference is that tuning the controller amounts to adjusting weighting kernels in LQR or adjusting the target system in backstepping.

\section{OPTIMAL LINEAR ESTIMATOR RESULTS}

\section{A. Problem Statement}

In the second part of the paper, we study the estimation problem which considers the following linear parabolic diffusion-reaction PDE system:

$$
\begin{aligned}
u_{t}(x, t) & =u_{x x}(x, t)+c u(x, t)+w(x, t) \\
u_{x}(0, t) & =0 \\
u(1, t) & =U(t) \\
u(x, 0) & =u_{0}(x) \\
y(t) & =u(0, t)+v(t)
\end{aligned}
$$

which adds a single measurement at the boundary $x=0$ with measurement noise $v(t)$. Moreover, the plant dynamics contain spatially distributed process noise $w(x, t)$ which represents model uncertainty. The covariance kernels for process and measurement noise are $W(x, y)$ and $V$, respectively. The initial state is also unknown and modeled as a zero mean random variable with covariance $S_{0}(x, y)$. Our goal is to design an optimal boundary measurement observer that estimates the state variable throughout the domain, in the presence of process and measurement noise.

The proposed linear estimator is as follows:

$$
\begin{aligned}
\hat{u}_{t}(x, t) & =\hat{u}_{x x}(x, t)+c \hat{u}(x, t)+L^{t}(y(t)-\hat{u}(0, t))(4) \\
\hat{y}(t) & =\hat{u}(0, t)
\end{aligned}
$$

with boundary conditions

$$
\hat{u}_{x}(0, t)=L_{0}^{t}(y(t)-\hat{u}(0, t)) \quad \hat{u}(1, t)=U(t)
$$

and initial condition

$$
\hat{u}(x, 0)=\hat{u}_{0}(x)
$$

where $L^{t}$ and $L_{0}^{t}$ are time-varying gains on the output estimation error. Namely, $L^{t}: C(0,1) \times \mathbb{R}^{+} \rightarrow \mathbb{R}$ is a continuous function over the spatial domain that weights the innovations and adds them to the dynamics. The gain $L_{0}^{t}: \mathbb{R}^{+} \rightarrow \mathbb{R}$ is scalar and adds weighted innovations to the boundary condition.

\section{B. Optimal Signal Injection}

As an intermediate step to solving the optimal estimator problem, we consider optimal signal injection. That is the output injection terms $L^{t}(y(t)-\hat{u}(0, t))$ and $L_{0}^{t}(y(t)-$ $\hat{u}(0, t))$ are replaced with arbitrary signals $l(x, t)$ and $l_{0}(t)$, respectively. We now design these signals to minimize the following quadratic cost criteria

$$
\begin{aligned}
J= & \mathrm{E}\left\{\frac{1}{2}\left\langle\tilde{u}(x, T), S_{0}(\tilde{u}(x, T))\right\rangle\right. \\
& +\frac{1}{2} \int_{0}^{T}[\langle\tilde{u}(x), W(\tilde{u}(x))\rangle] d t \\
& \left.+\frac{1}{2} \int_{0}^{T}\left[\langle l(x), V l(x)\rangle+V l_{0}^{2}\right] d t\right\}
\end{aligned}
$$

where $\tilde{u}(x, t)=u(x, t)-\hat{u}(x, t)$ and represents the state estimation error. This formulation mimics the open loop control problem in Section III-B. Here the observer error states are analogous to the regulated states, the injected signals are analogous to the control inputs, and the initial state uncertainty is analogous to the final desired state. Also note that the cost involves the expectation operator, since the states are stochastic due to process and measurement noise. In essence, this cost function optimally balances model uncertainty with measurement accuracy. Then our immediate goal is to determine the first-order necessary conditions for the injection signals which minimize the cost (45).

Lemma 4: Consider the linear diffusion-reaction PDE that describes the estimation error dynamics $(\tilde{u}(x, t)=u(x, t)-$ $\hat{u}(x, t))$ defined on the finite-time horizon $t \in[0, T]$ with quadratic cost criteria (45). Assume the covariance kernels $W(x, y), V(t), S_{0}(x, y)$ are zero-mean, Gaussian, and mutually independent. Replace the output injection terms $L^{t}(y(t)-\hat{u}(0, t))$ and $L_{0}^{t}(y(t)-\hat{u}(0, t))$ with the injection signals $l(x, t)$ and $l_{0}(t)$. Let $\tilde{u}^{*}(x, t), l^{*}(x, t), l_{0}^{*}(t)$, and $\lambda(x, t)$ respectively denote the optimal error state, dynamics injection signal, boundary injection signal, and co-state that minimize the quadratic cost (45). Then the first order necessary conditions for optimality are:

$$
\begin{aligned}
\tilde{u}_{t}^{*}(x, t) & =\tilde{u}_{x x}^{*}(x, t)+c \tilde{u}^{*}(x, t)-l^{*}(x, t) \\
-\lambda_{t}(x, t) & =\lambda_{x x}+c \lambda(x, t)+W\left(\tilde{u}^{*}(x, t)\right)
\end{aligned}
$$

with boundary conditions

$$
\begin{aligned}
& u_{x}^{*}(0, t)=-l_{0}^{*}(t) \quad u^{*}(1, t)=0 \\
& \lambda_{x}(0, t)=0 \quad \lambda(1, t)=0
\end{aligned}
$$


(a)

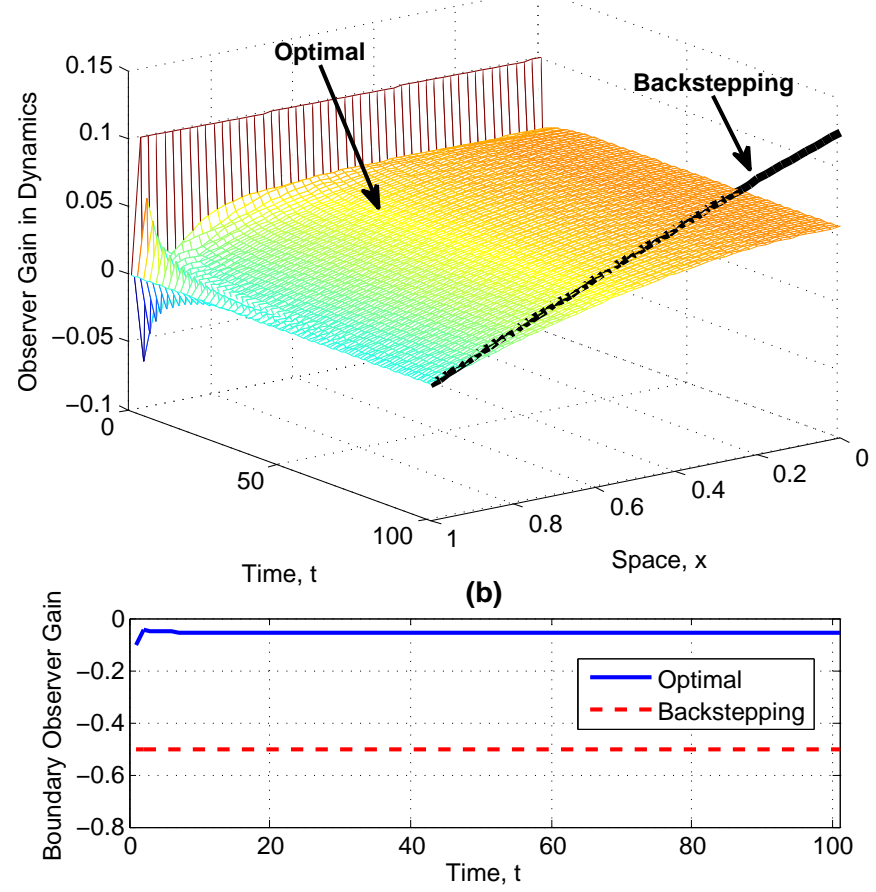

Fig. 3. Observer gains for the (a) dynamics and (b) boundary for the optimal and backstepping estimators.

and split initial/final conditions

$$
\tilde{u}^{*}(x, 0)=\tilde{u}_{0}(x) \quad \lambda(x, T)=S_{0}\left(\tilde{u}^{*}(x, T)\right)
$$

and the optimal injection signals are

$$
l^{*}(x, t)=\frac{1}{V} \lambda(x, t) \quad l_{0}^{*}(t)=-\frac{1}{V} \lambda(0, t)
$$

Proof: The necessary conditions are derived via weak variations [18] using the exact same approach as in Section III-B.

The optimal signal injection does not provide a practical implementation approach to the observer problem. Indeed, the goal here is to provide an intermediate step to the next result.

\section{Output Injection}

Now we consider output injection. Namely, we postulate that the co-state for the optimal signal injection problem is related to the estimator's output error according to the following time-varying linear transformation:

$$
\lambda(x, t)=\int_{0}^{1} S(x, y, t) \delta(y) \tilde{u}(y, t) d y=S(x, 0, t) \tilde{u}(0, t)
$$

where $\delta(y)$ is the Dirac delta function used to sift out the boundary value where the sensor is located.

Theorem 5: The optimal gains for $L^{t}$ and $L_{0}^{t}$ can be found by solving the following dual Riccati PDE:

$$
\begin{aligned}
S_{t} & =S_{x x}+2 c S-\frac{1}{V} S(x, t) S(0, t)+W(x, 0)(53) \\
S(1, t) & =S_{x}(0, t)=0 \\
S(x, 0) & =S_{0}(x, 0)
\end{aligned}
$$

which are related to the output injection gains according to

$$
\begin{aligned}
L^{t} & =\frac{1}{V} S(x, t) \\
L_{0}^{t} & =-\frac{1}{V} S(0, t)
\end{aligned}
$$

Proof: The proof consists of evaluating each $\lambda$ term in (47), (49), and (50) using the postulated form in (52). In this derivation the sifting and derivative properties of the Dirac-delta function, described in Section II, are useful. As in the state-feedback control derivation, boundary conditions arise from (49) and integration by parts. This eventually produces a Riccati-type PDE that is solved backward in time. We rewrite this PDE by scaling time by a factor of -1 , which produces a PDE that is solved forward in time. Finally, we note that two boundary conditions arising from integration by parts and the derivative of the Dirac-delta function indicate that $S(x, y, t)$ has no variation across the $\mathrm{y}$ dimension. This reduces the PDE into one spatial and one temporal dimension, providing the result indicated above.

In an analogous situation to finite-dimensional optimal linear estimators, the dual-Riccati PDE (53)-(55) is solved forward in time, while the Riccati-PDE in (27)-(29) is solved backward in time. It is also interesting to note that the dualRiccati PDE is 1-D spatial and 1-D temporal, while the Riccati PDE is 2-D spatial and 1-D temporal. This difference is related to the fact that the domains of the state-feedback and output-injection operators are infinite-dimensional and scalar, respectively.

TABLE II

Parameter Values for Estimator Simulation Examples

\begin{tabular}{ll}
\hline \hline Parameter & Value \\
\hline \hline Reaction coefficient & $c=1$ \\
Process noise cov. kernel & $W(x, y)=10^{-1} \cdot I(x, y)$ \\
Measurement noise variance & $V=1$ \\
Initial state cov. kernel & $S_{0}(x, y)=0.1 \cdot I(x, y)$ \\
Exogenous input & $U(t)=0.5+0.1 \sin (2 \pi t)$ \\
Initial plant state & $u(x, 0)=0.3 \quad \forall x$ \\
Initial observer state & $\hat{u}(x, 0)=0.1 \quad \forall x$ \\
Time Horizon & $T=1$ \\
\hline \hline
\end{tabular}

\section{Simulation Example}

Here we demonstrate the optimal linear estimator results, where the optimal output injection gains are given by (56) and (57), and the time-varying linear operator $S(x, t)$ is the unique solution of the dual Riccati PDE (53)-(55). The parameters for this example are provided in Table II. The optimal output injection gains are shown graphically in Fig. 3. The initial gain is proportional to the covariance 
(a) Optimal Estimator

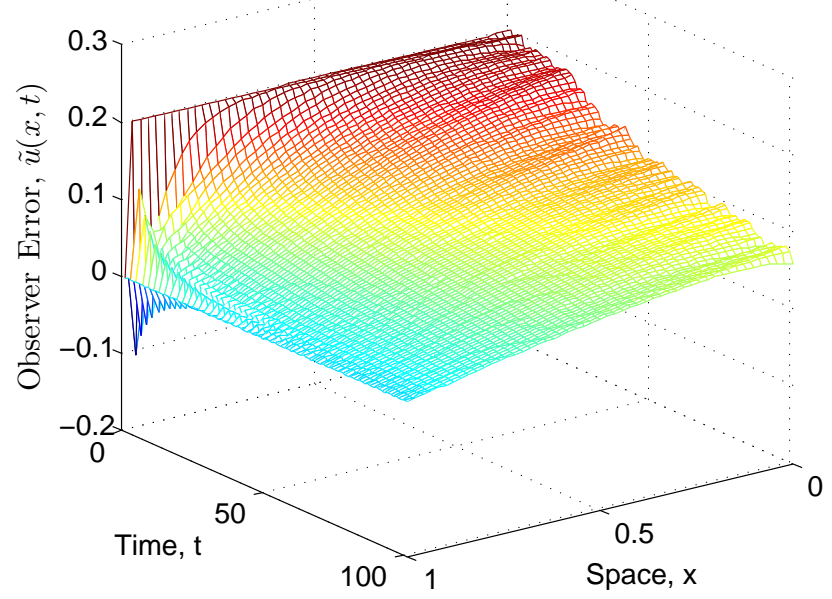

(b) Backstepping Estimator

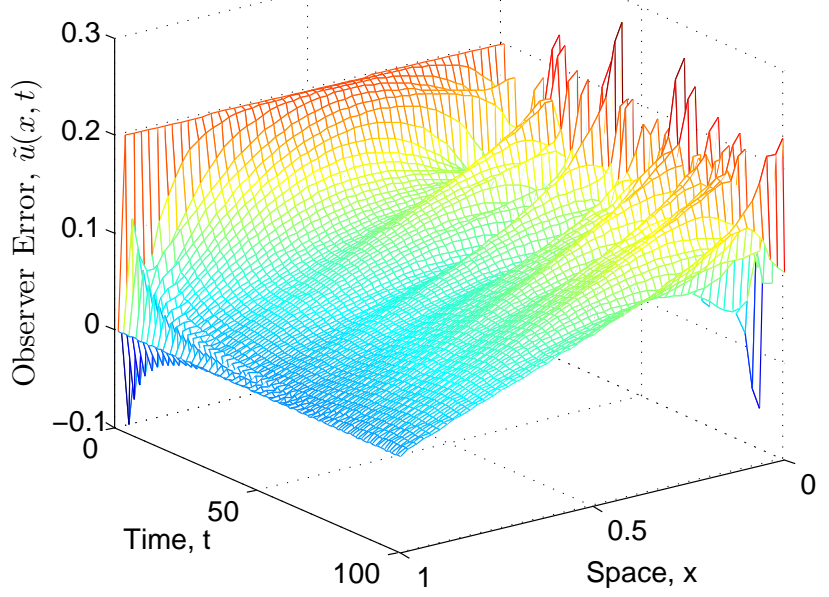

Fig. 4. Simulation example of state estimation error trajectories for the (a) optimal linear and (b) backstepping observers.

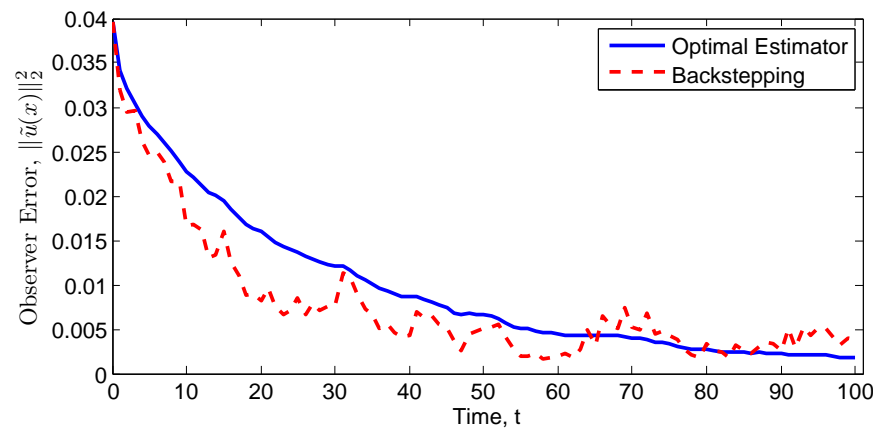

Fig. 5. L2-norm of estimation error for optimal linear and backstepping observers.

kernel for the initial state uncertainty. In simulation the noise covariance kernels used to tune the gains are also applied on the plant itself. An exogenous input $U(t)$ given by the expression in Table II is also applied to the system. Finally, we intentionally initialize the plant and observer at different initial states (see Table II).

Simulation results for the estimation error are provided in Fig. 4(a), which approaches zero as time increases. This fact can also be seen in the $\mathrm{L}^{2}$-norm of the estimation error, shown graphically in Fig. 5. Thus, the proposed optimal estimator converges toward the true state.

\section{E. Comparison to Backstepping}

In the estimation problem, the backstepping observer takes the exact same form as the linear optimal estimation described in (41)-(44). The backstepping procedure renders the estimation error dynamics into the heat equation target system. The authors of [22] demonstrated that the observer gains are related to the solution of the following hyperbolic Klein-Gordon PDE:

$$
p_{x x}(x, y)-p_{y y}(x, y)=c p(x, y)
$$

$$
\begin{array}{r}
p(x, x)=\frac{1}{2} c(x-1) \\
p(1, y)=0
\end{array}
$$

solved on the region $\{x, y: 0<y<x<1\}$ where $L(x)=$ $p_{y}(x, 0)$ and $L_{0}=p(0,0)$. Using a summation of successive approximation series, one can show that the solution to this PDE is

$$
p(x, y)=-c(1-x) \frac{I_{1}(\sqrt{c(2-x-y)(x-y)})}{\sqrt{c(2-x-y)(x-y)}}
$$

where $I_{1}$ is the first-order modified Bessel function of the first kind. Then the backstepping observer gains are given by

$$
\begin{aligned}
L(x) & =\frac{c(1-x)}{x(2-x)} I_{2}(\sqrt{c x(2-x)}) \\
L_{0} & =-\frac{c}{2}
\end{aligned}
$$

A comparison of the time-varying optimal observer gains and time-invariant backstepping observer gains are shown in Fig. 3. In general, these figures indicate that the backstepping observer provides more aggressive corrections to errors in the predicted output, both in the dynamics and at the boundary. This makes the backstepping observer more sensitive to noise, however. Both of these properties are seen in the trajectory of the observer error shown in Fig. 4(b), adjacent to the optimal estimator results. In particular, the output injection applied at the boundary condition $u_{x}(0, t)$ for backstepping results in significantly noisier estimates relative to the optimal observer. Nonetheless, the overall estimation error measured in terms of the $\mathrm{L}^{2}$-norm across the spatial domain in Fig. 5 indicates that the optimal and backstepping observers have similar convergence rates. One may desensitize the backstepping estimator to noise by adjusting the target system, as in the control problem. Nonetheless we see that the optimal estimator compares favorably with backstepping. The key differences in each approach are in the design criteria. The optimal estimator 
is tuned by adjusting the noise covariance kernels whereas the backstepping observer is tuned by adjusting the target system.

\section{CONCLUSIONS}

This paper presents methods for optimal control and optimal estimation of linear parabolic PDEs characterized by diffusion-reaction dynamics and actuators/sensors at the boundaries. The focus is limited to Dirichlet actuation and anti-collocated actuators/sensors. Through optimal control techniques first order necessary conditions are derived for both the optimal control and optimal estimation problems. In the control case, both open loop and state-feedback results are presented. In the estimation case, open loop signal injection provides an intermediate step to obtain output injection results. In both cases the optimal linear transformation kernels are governed by Riccati-like partial differential equations, which have clear connections to the traditional Riccati differential equations for finite-dimensional systems and the operator Riccati equations from semigroup theoretical techniques for infinite-dimensional systems. These results are elegant, computationally tractable, and intuitive to tune. Finally, numerical examples and a comparative analysis to the established backstepping approach demonstrate the results presented here.

\section{ACKNOWLEDGMENTS}

The authors would like to thank the National Science Foundation Graduate Research Fellowship Program (NSF GRFP) for their financial support.

\section{REFERENCES}

[1] G. Avalos and I. Lasiecka, "Differential Riccati equation for the active control of a problem in structural acoustics," Journal of Optimization Theory and Applications, vol. 91, no. 3, pp. 695 - 728, 1996.

[2] I. Aksikas, A. Fuxman, J. F. Forbes, and J. J. Winkin, "Lq control design of a class of hyperbolic pde systems: Application to fixed-bed reactor," Automatica, vol. 45, no. 6, pp. 1542-1548, 2009.

[3] G. Ferrari-Trecate, A. Buffa, and M. Gati, "Analysis of coordination in multi-agent systems through partial difference equations," IEEE Transactions on Automatic Control, vol. 51, no. 6, pp. 1058 - 1063, 2006.

[4] T. Zariphopoulou, "Optimal investment and consumption models with non-linear stock dynamics," Mathematical Methods of Operations Research, vol. 50, no. 2, pp. 271 - 96, 1999.

[5] R. Vazquez and M. Krstic, "Explicit output feedback stabilization of a thermal convection loop by continuous backstepping and singular perturbations," in 2007 American Control Conference, 2007 2007, pp. 2177-2182.

[6] S. Dubljevic, "Optimal boundary control of cardiac alternans," International Journal of Robust and Nonlinear Control, vol. 19, no. 2, pp. 135-50, January 2009.

[7] D. Del Vecchio and N. Petit, "Boundary control for an industrial underactuated tubular chemical reactor," Journal of Process Control, vol. 15, no. 7 , pp. $771-784,2005$.
[8] M. Doyle, T. Fuller, and J. Newman, "Modeling of galvanostatic charge and discharge of the lithium/polymer/insertion cell," Journal of the Electrochemical Society, vol. 140, no. 6, pp. 1526 - 33, 1993.

[9] T. Fuller, M. Doyle, and J. Newman, "Simulation and optimization of the dual lithium ion insertion cell," Journal of the Electrochemical Society, vol. 141, no. 1, pp. 1 - 10, 1994.

[10] J. C. Forman, S. Bashash, J. L. Stein, and H. K. Fathy, "Reduction of an electrochemistry-based li-ion battery degradation model via constraint linearization and pade approximation," accepted to Journal of the Electrochemical Society, 2010.

[11] A. Bensoussan, M. C. Delfour, and S. K. Mitter, "The linear quadratic optimal control problem for infinite dimensional systems over an infinite horizon; survey and examples," in Proceedings of the IEEE Conference on Decision and Control, Dec. 1976, pp. 746-51.

[12] D. Di Domenico, A. Stefanopoulou, and G. Fiengo, "Lithium-ion battery state of charge and critical surface charge estimation using an electrochemical model-based extended Kalman filter," Journal of Dynamic Systems, Measurement and Control, Transactions of the ASME, vol. 132, no. 6, 2010.

[13] G. Lube and B. Tews, "Optimal control of singularly perturbed advection-diffusion-reaction problems," Mathematical Models and Methods in Applied Sciences, vol. 20, no. 3, pp. 375 - 395, 2010.

[14] S. Ravindran, "A reduced-order approach for optimal control of fluids using proper orthogonal decomposition," International Journal for Numerical Methods in Fluids, vol. 34, no. 5, pp. 425 - 48, 2000.

[15] A. Bensoussan, Representation and control of infinite dimensional systems. Birkhauser, 1992.

[16] R. F. Curtain and H. J. Zwart, An introduction to infinite-dimensional linear systems theory. Springer, 1995.

[17] I. Lasiecka and R. Triggiani, Control theory for partial differential equations: continuous and approximation theories. Cambridge University Press, 2000.

[18] D. S. Bernstein and P. Tsiotras, A Course in Classical Optimal Control. Pre-print, 2009

[19] R. L. Burden and J. D. Faires, "Numerical analysis (7th)," Prindle Weber and Schmidt, Boston, 2001.

[20] M. Krstic and A. Smyshlyaev, Boundary control of PDEs: A Course on Backstepping Designs. SIAM Advances in Design and Control series, 2008.

[21] A. Smyshlyaev and M. Krstic, "Closed-form boundary state feedbacks for a class of 1-d partial integro-differential equations," IEEE Transactions on Automatic Control, vol. 49, no. 12, pp. 2185-202, 122004.

[22] _ - "Backstepping observers for a class of parabolic pdes," System and Control Letters, vol. 54, no. 7, pp. 613-625, 2005. 